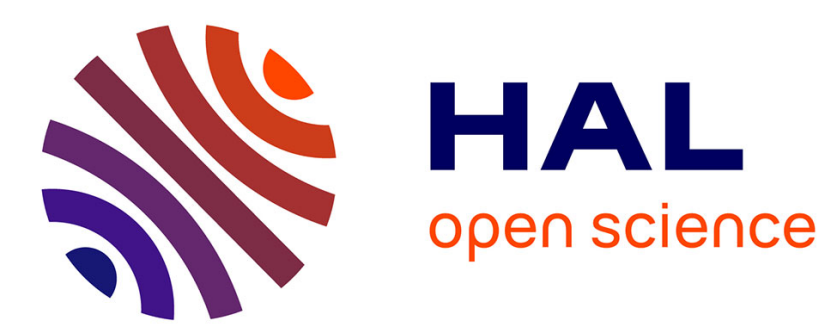

\title{
Nouvelle technique de marquage d'écoulement par utilisation de molécules photochromes
}

\author{
A. d'Arco, J.C. Charmet, M. Cloitre
}

\section{To cite this version:}

A. d'Arco, J.C. Charmet, M. Cloitre. Nouvelle technique de marquage d'écoulement par utilisation de molécules photochromes. Revue de Physique Appliquée, 1982, 17 (2), pp.89-93. 10.1051/rphysap:0198200170208900 . jpa-00244976

\section{HAL Id: jpa-00244976 https://hal.science/jpa-00244976}

Submitted on 1 Jan 1982

HAL is a multi-disciplinary open access archive for the deposit and dissemination of scientific research documents, whether they are published or not. The documents may come from teaching and research institutions in France or abroad, or from public or private research centers.
L'archive ouverte pluridisciplinaire HAL, est destinée au dépôt et à la diffusion de documents scientifiques de niveau recherche, publiés ou non, émanant des établissements d'enseignement et de recherche français ou étrangers, des laboratoires publics ou privés. 


\title{
Nouvelle technique de marquage d'écoulement par utilisation de molécules photochromes
}

\author{
A. D'Arco, J. C. Charmet et M. Cloitre \\ Laboratoire d'Hydrodynamique et Mécanique Physique, E.S.P.C.I., 10, rue Vauquelin, 75005 Paris, France
}

(Reçu le 4 septembre 1981, accepté le 3 novembre 1981)

\begin{abstract}
Résumé. - Poursuivant la réalisation de méthodes de caractérisation d'un fluide en écoulement par diffusion Rayleigh forcée, nous avons mis au point une technique de marquage d'une figure bidimensionnelle quelconque dans le fluide par imagerie optique.

L'impression de ce réseau et la visualisation directe de sa déformation permettent d'obtenir une mesure directe des composantes du gradient de vitesse.
\end{abstract}

\begin{abstract}
In a series of studies of the characterization of a flowing liquid based on the forced Rayleigh scattering technique, we have written bidimensional gratings in the fluid by using direct optical imaging.

The writing of this grid and the direct visualization of its deformation permit in particular a direct measurement of the components of the velocity gradient.
\end{abstract}

Introduction. - Les techniques de diffusion Rayleigh forcée ont été récemment appliquées à l'étude des écoulements hydrodynamiques laminaires et turbulents [1]. Dans ces expériences, le fluide est marqué thermiquement par une figure périodique de pas $p$ obtenue par interférence de deux faisceaux issus d'un laser pulsé puissant (montage optique identique à celui d'un anémomètre laser Doppler). Le liquide contient un colorant, par exemple du rouge de méthyle, dont le rôle est d'élever le coefficient d'absorption à la longueur d'onde de marquage.

L'écriture doit être suffisamment rapide pour que le fluide se déplace d'une quantité très inférieure au pas $p$ pendant le temps de l'impulsion laser, ceci afin d'assurer la bonne netteté du réseau. La modulation spatiale de température résultant de l'absorption du faisceau d'écriture se traduit par une modulation d'indice de réfraction. Le réseau de phase ainsi réalisé est convecté par l'écoulement.

L'évolution de ce réseau est suivie par diffraction d'un faisceau laser auxiliaire, conduisant à des mesures de diffusivité, de vitesse et de gradient de vitesse.

a) Diffusivité [2]. - Les mécanismes de diffusion de la chaleur sont responsables de l'homogénéisation de la température au sein du fluide, donc de la relaxation de la modulation d'indice, ce qui induit de façon corrélée une relaxation de l'intensité des ordres diffractés par le réseau.

L'évolution temporelle de la figure de marquage initialement représentée par une modulation sinusoïdale de température :

$$
\theta(x, t=0)=\theta_{0} \sin k x,
$$

avec $k$ vecteur d'onde du réseau $(2 \pi / p)$ est régie par l'équation de Fourier de la chaleur :

$$
\frac{\partial \theta}{\partial t}=D \Delta \theta
$$

$D$ étant le coefficient de diffusivité thermique du liquide.

Elle admet pour solution :

$$
\theta(t)=\theta(t=0) \exp \left(-D k^{2} t\right)
$$

ce qui conduit à la loi de décroissance exponentielle de l'intensité diffractée dans le premier ordre de diffraction :

$$
I \sim \exp (-2 t / \tau)
$$

$\tau$ représente un temps caractéristique de persistance du réseau et a pour valeur :

$$
\tau=p^{2} / 4 \pi^{2} D .
$$


Il est d'environ $1 / 4 \mathrm{~s}$ pour un réseau de pas $1 \mathrm{~mm}$, le coefficient de diffusivité des liquides étant de l'ordre de $D=10^{-3} \mathrm{~cm}^{2} / \mathrm{s}$.

b) VitesSe [3]. - Le défilement du réseau à la vitesse moyenne $V$ devant le faisceau de lecture affecte les ordres diffractés d'un décalage Doppler de fréquence optique $\Delta f=V / p$.

En recevant simultanément sur un photodétecteur un ordre diffracté et une fraction de l'ordre zéro non affecté du décalage Doppler, le signal électrique présente, portée par la loi de décroissance exponentielle due à la diffusion, une modulation périodique à la fréquence $\Delta f$. Ce battement temporel est identique à celui délivré par les anémomètres Doppler à un faisceau diffusé (anémométrie homodyne); le réseau d'indice joue le rôle des particules diffusantes.

c) Gradients de vitesse [4]. - Dans de nombreux écoulements laminaires et turbulents, c'est la variation de vitesse d'un point à l'autre, son gradient, qui en contrôle les propriétés. L'analyse du déplacement des ordres diffractés dans le plan de Fourier conduit à la mesure de deux composantes du gradient de vitesse. Nous éliminons la vitesse moyenne d'écoulement par suite de la propriété d'invariance par translation de la transformée de Fourier. Dans un référentiel $O x_{1} x_{2}$, $O x_{1}$ étant l'axe perpendiculaire aux traits du réseau à l'instant initial et $O x_{2}$ l'axe parallèle, le gradient élongationnel $\partial v_{1} / \partial x_{1}$ se traduit par une variation du pas du réseau, donc par une variation d'écartement entre les ordres diffractés, tandis que le gradient transverse $\partial v_{1} / \partial x_{2}$ entraîne le réseau en rotation dans son plan et, par conséquent, donne une rotation identique de la figure de diffraction.

Cependant, le marquage par interférence de faisceaux laser se prête mal à une géométrie autre que celle d'un réseau et en particulier au marquage d'une grille carrée nécessaire à la détermination des deux autres composantes du gradient $\partial v_{2} / \partial x_{1}$ et $\partial v_{2} / \partial x_{2}$, qui permettent une détermination complète de la déformation d'une particule fluide dans un plan. Ces raisons nous ont conduit à mettre au point une nouvelle technique de marquage d'une figure géométrique plane quelconque grâce à un procédé d'imagerie classique.

1. Technique de marquage par imagerie optique [5]. - La figure 1 représente l'ensemble du dispositif optique. La source de lumière pulsée (flash incohérent pour appareil photo Mecablitz CT de nombre guide 45) éclaire une grille objet GO dont le système optique OP forme l'image dans la cellule d'écoulement $\mathrm{C}$. Le motif du marquage peut alors être choisi à volonté; une grille carrée constitue une géométrie adéquate pour la mesure des gradients. La grande souplesse du montage permet de faire varier le pas de la grille image de $50 \mu \mathrm{m}$ à plusieurs millimètres.

Cependant, une limitation est fixée par la profondeur de champ de l'image. Celle-ci conditionne l'épaisseur de l'image marquée et, par voie de consé-

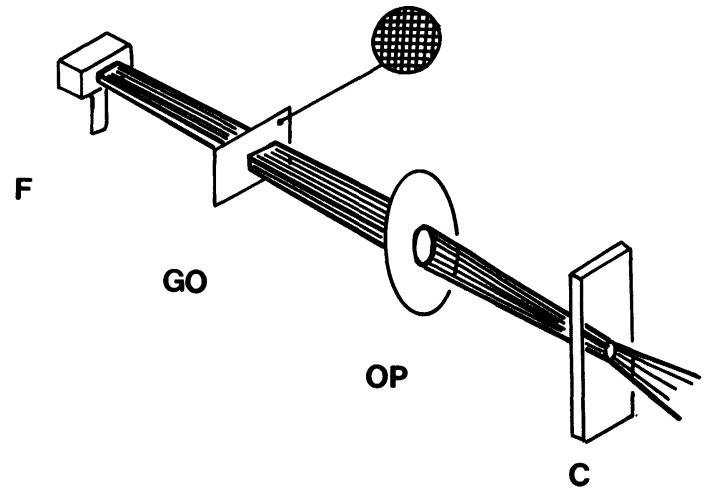

Fig. 1. - Vue schématique du dispositif d'imagerie optique. F : flash d'écriture. GO : grille objet. OP : objectif photographique $(50 \mathrm{~mm} \mathrm{f} / 1,2)$. $\mathrm{C}$ : cellule d'écoulement.

[Experimental set up of the optical imagery apparatus. $\mathrm{F}$ : writing flash. GO : source grid. OP : photographic lens (50 $\mathrm{mm} \mathrm{f} / 1.2)$. C : flow cell.]

quence, la précision des mesures hydrodynamiques par suite de l'intégration sur l'épaisseur du réseau des distorsions induites par l'écoulement. La profondeur de champ $S$ dépend du paramètre d'ouverture numérique de l'optique $f=F / \varphi$ ( $F$ est la distance focale de l'objectif, $\varphi$ son diamètre), du pas $p$ de la trame image et du grandissement $m$ du système. Tant que la trame est résolue par le système optique, $S$ se calcule dans l'approximation de l'optique géométrique (Fig. 2) et s'exprime par la relation :

$$
S=2 p(m+1) f
$$

dans le domaine paraxial.

Le minimum de $S$ est fixé par la limite de diffraction lorsque le pas $p$ devient de l'ordre de grandeur de la tache d'Airy image $\lambda(m+1) f$ et vaut alors

$$
S_{\mathrm{L}}=2 \lambda(m+1)^{2} f^{2} \quad[5] \text {. }
$$

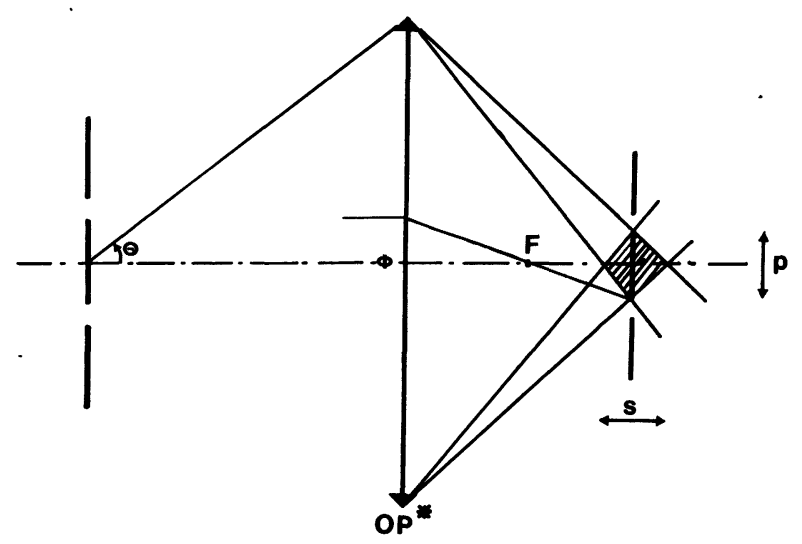

Fig. 2. - Vue schématique de la grille image. $\mathrm{S}$ : profondeur de champ. $p:$ pas du réseau.

[Shematic of the image grid. $S:$ field depth. $p:$ interfrange spacing.] 
Il y a donc tout intérêt à utiliser une optique de grande ouverture numérique ( $f$ petit) et un grandissement aussi faible que possible. Cependant, l'obligation de rester dans le domaine paraxial et de collecter la lumière émise par le flash dans l'angle solide le plus étendu possible impose un compromis sur les valeurs tolérables du grandissement $m$.

Le tableau I donne les valeurs du rapport $S / p$ et de l'angle de collection $\Theta$ en fonction de $m$ pour un objectif photo Nikon $50 \mathrm{mmf} / 1,2$.

Tableau I

\begin{tabular}{|c|c|c|c|c|c|}
\hline $\mathrm{m}$ & $1 / 5$ & $1 / 4$ & $1 / 3$ & $1 / 2$ & 1 \\
\hline $\mathrm{s} / \mathrm{p}$ & 0.58 & 0.75 & 1.1 & 1.8 & 4.8 \\
\hline$\theta$ & $8^{\circ}$ & $9.5^{\circ}$ & $12^{\circ}$ & $16^{\circ}$ & $23.5^{\circ}$ \\
\hline
\end{tabular}

2. Marquage chimique. - Utilisation de molécules photochromes.

Lors d'un marquage thermique, la lecture par un faisceau laser auxiliaire s'effectue soit par analyse de la figure de diffraction de la grille de phase, soit par visualisation de cette grille sous forme d'une image d'amplitude par strioscopie, ce qui nécessite dans les deux cas une séparation des ordres diffractés, donc un pas image inférieur au mm. Au-delà, il est nécessaire de marquer une figure d'amplitude plutôt qu'une figure de phase.

Pour ce faire, nous avons utilisé une solution de colorant photochrome spiropyranne (nitro-indolinospyranne) dans du toluène. Sous l'action du rayonnement proche U.V. du flash d'écriture, la solution initialement jaune pâle passe dans un état excité de

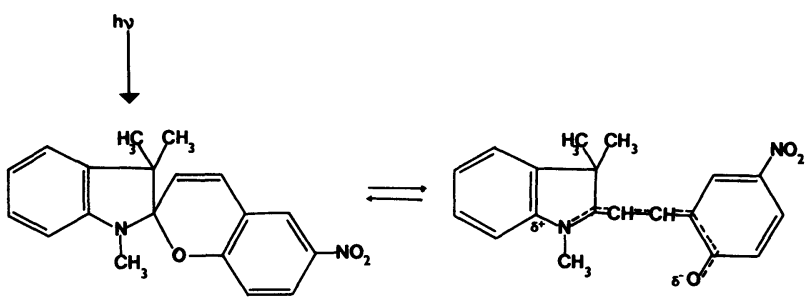

Fig. 3. - Photochimie du 6 nitro-indolinospyranne.

[Photochemistry of the 6 nitro-indolinospyranne.] couleur bleu sombre par rupture de la liaison $\mathrm{C}-\mathrm{O}$ du cycle pyrannique [6] (Fig. 3).

On réalise ainsi un marquage d'amplitude par la présence de deux espèces présentant une différence d'absorbance importante. Indépendamment de la relaxation par diffusion massique caractérisée comme en marquage thermique par le temps de demi-persistance $\tau=p^{2} / 4 \pi^{2} D(D$ coefficient de diffusion massique est environ cent fois plus faible que le coefficient de diffusion de la chaleur; l'équation de Fourier est remplacée par l'équation de Fick de diffusion de masse), la désexcitation se produit spontanément par effet thermique ou sous l'action photochimique d'une lumière au bout d'un temps de l'ordre de $10 \mathrm{~s}$ pour une concentration de $10^{-2}$ à $10^{-4}$ moles $/$ l.

Dans tous les cas, ce marquage se révèle favorable à l'analyse des gradients par suite de l'augmentation des temps caractéristiques de relaxation par rapport au marquage thermique.

3. Résultats préliminaires. - 3.1 EXEMPLE DE VISUALISATION. - De par la géométrie plane de l'image, la technique décrite est bien adaptée à l'étude des écoulements bidimensionnels type Hele Shaw et, plus généralement, à celle des écoulements à proximité d'une paroi transparente.

Les photographies de la figure 4 présentent un exemple de son utilisation pour visualiser la déformation d'un réseau et d'une grille carrée de pas millimétrique sous l'action d'un mouvement de convection thermique déclenché par chauffage d'une face latérale de la cuve de dimension $1 \mathrm{~mm} \times 10 \mathrm{~mm} \times 50 \mathrm{~mm}$. L'observation de la figure déformée par le champ de vitesse représentée en figure 5 est réalisée 1 seconde après le marquage.

3.2 DÉTERMINATION DES GRADIENTS. - L'information contenue dans la photographie de la grille carrée est très riche puisque l'on dispose en chaque point de l'image de la figure de distorsion d'un carré élémentaire, ce qui permet de remonter aux valeurs des gradients élongationnels et de la vorticité locale.

En effet, abstraction faite de la translation d'ensemble à la vitesse du centre du carré, la distorsion induite par l'écoulement est un cisaillement simple dont le champ de déformation se décompose en trois termes :

$$
\left|\begin{array}{ll}
\frac{\partial u_{1}}{\partial x_{1}} & \frac{\partial u_{1}}{\partial x_{2}} \\
\frac{\partial u_{2}}{\partial x_{1}} & \frac{\partial u_{2}}{\partial x_{2}}
\end{array}\right|=\left|\begin{array}{cc}
\frac{\partial u_{1}}{\partial x_{1}} & 0 \\
0 & \frac{\partial u_{2}}{\partial x_{2}}
\end{array}\right|+\frac{1}{2} \mid \begin{gathered}
0 \\
\frac{\partial u_{1}}{\partial x_{2}}+\frac{\partial u_{2}}{\partial x_{1}} \\
\begin{array}{c}
\text { dilatation } \\
\text { (de trace nulle) }
\end{array}
\end{gathered}
$$

$$
\begin{gathered}
\frac{\partial u_{1}}{\partial x_{2}}+\frac{\partial u_{2}}{\partial x_{1}}\left|+\frac{1}{2}\right| \begin{array}{cc}
0 & -\frac{\partial u_{1}}{\partial x_{2}}-\frac{\partial u_{2}}{\partial x_{1}} \\
0 & \mid \frac{\partial u_{1}}{\partial x_{2}}+\frac{\partial u_{2}}{\partial x_{1}}
\end{array} \\
\text { élongation }
\end{gathered}
$$

u est le vecteur déplacement. 

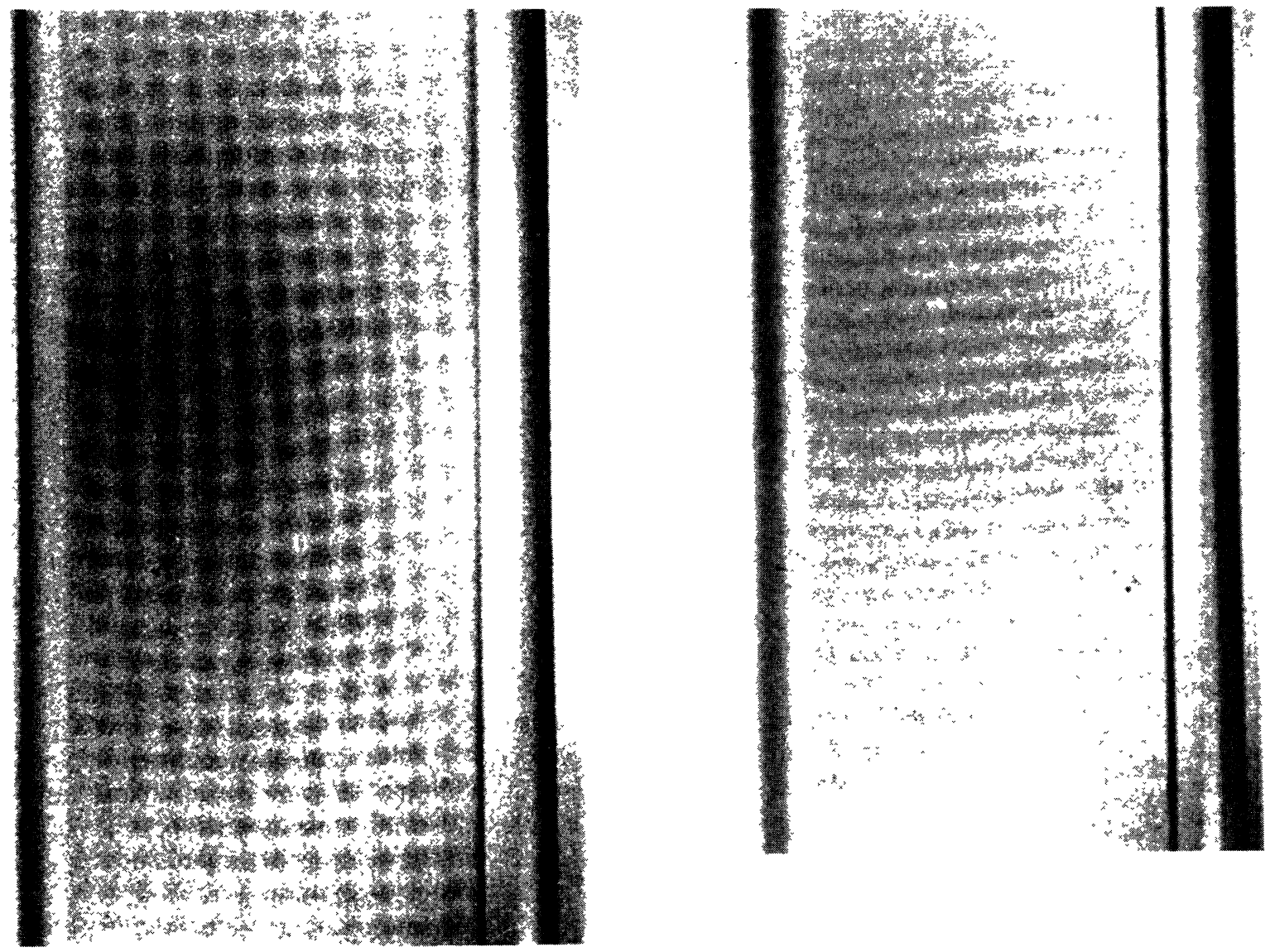

Fig. 4. - Visualisation de la grille image déformée par un écoulement de convection thermique.

[Visualization of the image grid distorted by a thermal convective flow.]

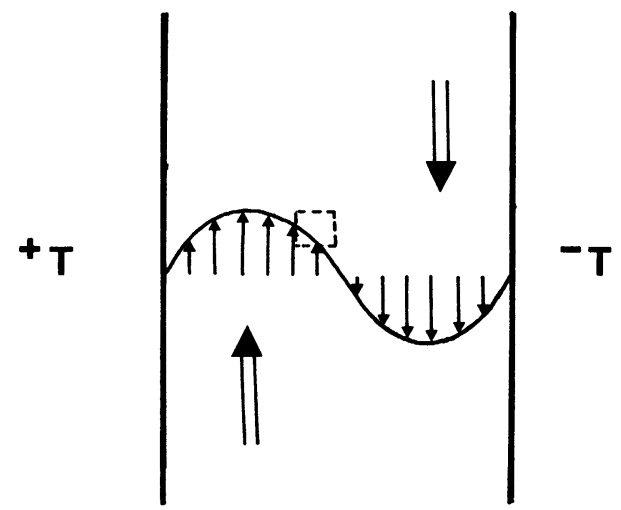

Fig. 5. - Allure du champ de vitesse dans un écoulement de convection libre.

[Velocity field in a free convection flow.]

Le deuxième terme s'exprime dans les axes propres $X_{1} X_{2}$ sous la forme :

$$
\frac{1}{2}\left[\begin{array}{cc}
\partial u_{2} / \partial X_{1}+\partial u_{1} / \partial X_{2} & 0 \\
0 & -\left(\partial u_{2} / \partial X_{1}+\partial u_{1} / \partial X_{2}\right)
\end{array}\right]
$$

caractéristique d'une élongation.
Cette décomposition est schématisée sur la figure 6 . La comparaison des photographies au temps $t=0$ et au temps $t=\delta t$ donne accès au champ de déformation $\partial u_{\mathrm{i}} / \partial x_{\mathrm{j}}$ donc au champ des gradients de vitesse $\partial v_{\mathbf{i}} / \partial x_{\mathbf{j}}:$

$$
\frac{\partial u_{\mathbf{i}}}{\partial x_{\mathbf{j}}}=\frac{\partial v_{\mathbf{i}}}{\partial x_{\mathbf{j}}} \delta t
$$

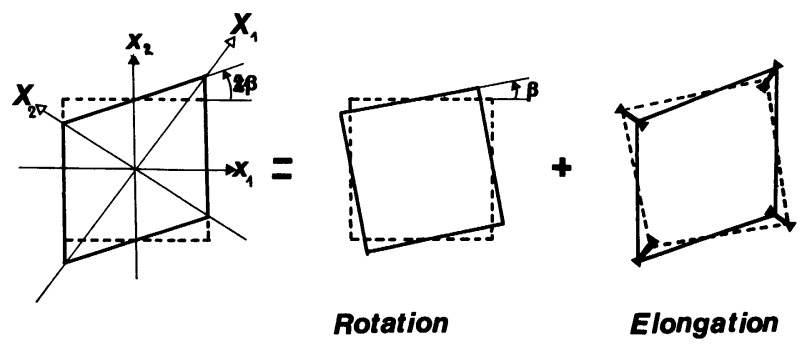

Fig. 6. - Décomposition d'un champ de déformation.

[Analysis of a deformation field.]

En réalité, cette mesure procède à une double moyenne spatiale et temporelle. La déformation du carré élémentaire est représentative de la valeur 
moyenne du gradient sur un demi pas de la grille et de la valeur moyenne sur l'intervalle de temps $\delta t$. Cette dernière moyenne n'affecte pas le résultat en écoulement permanent, par contre elle ne peut plus être représentative de l'histoire des gradients en écoulement turbulent : le choix de l'intervalle $\delta t$ de lecture-écriture devra alors être optimisée en fonction des caractéristiques de l'écoulement.

3.3 SENSIBILITÉ DE LA MÉTHODE. - Il faut estimer dans la formule $\partial u_{\mathrm{i}} / \partial x_{\mathrm{j}}=\partial v_{\mathrm{i}} / \partial x_{\mathrm{j}} \delta t$ les gradients minimum mesurables compte tenu du plus petit déplacement repérable sur les visualisations obtenues. L'erreur de mesure est assimilable à la définition du bord de trait, elle-même fixée par le système optique d'écriture : c'est la largeur de la tâche d'Airy dans le plan image soit : $\lambda(m+1) f$. La plus petite valeur de gradient de vitesse détectable est alors donnée par :

$$
2(m+1) f /(p \mathrm{~d} t) .
$$

Dans le cas de l'expérience où nous utilisons un objectif Nikon $50 \mathrm{~mm}$ avec une ouverture 1,2 au grandissement 1 , un pas millimétrique, nous avons :

$$
\partial t \sim 1 \mathrm{~s} .
$$

Et la sensibilité en gradient de vitesse est : $2 \times 10^{-3} \mathrm{~s}^{-1}$.

4. Conclusion. - Cette méthode simple et peu perturbative permet de visualiser le champ de déformation du liquide dans un écoulement à différentes échelles, fonctions du pas de la grille.

Elle donne accès aux gradients élongationnels dans le plan image et à une mesure de vorticité perpendiculaire à ce plan au moyen d'un procédé de marquage souple et peu coûteux, ce qui permet d'envisager notamment son utilisation dans un but pédagogique [7].

Par ailleurs, associée à un dispositif d'analyse d'image, elle permet la caractérisation des écoulements turbulents par l'analyse statistique des différentes composantes fluctuantes des gradients de vitesse, particulièrement celles qui sont responsables de l'étirement de la vorticité.

\section{Bibliographie}

[1] Fermigier, M., Cloitre, M., Guyon, E., JenfFer, P., Utilisation de la diffusion Rayleigh forcée à l'étude d'écoulements laminaires et turbulents. Soumis au Journal de Mécanique (1981).

[2] Fermigier, M., Mesures sur les écoulements laminaires et turbulents par marquage optique. Thèse de 3e cycle, Paris VI (1980).

[3] Fermigier, M., Jenffer, P., Charmet, J. C. et Guyon, E., A non-perturbative anemometric and flow visualization technique. J. Physique Lett. 41 (1980) 519-521.
[4] Fermigier, M., Guyon, E., Jenffer, P. et Petit, L., A direct optical measurement of velocity gradients. Appl. Phys. Lett. 36 (1980) 361-362.

[5] Brown, G. H., Photochromism. Techniques of chemistry (Wiley Interscience) Tome III, Chap. 1, 100 (1971).

[6] Colombeau, B., Froehly, C., Vampouille, M., Fourier description of the axial structure of speckle. J. Optics 10 (1979) 65-69.

[7] D'Arco, A., Cloitre, M., Guyon, E., Jenffer, P., Sur une mesure en laboratoire de travaux pratiques de diffusivité thermique et massique. Accepté à : Europ. J. Phys. 